\title{
Biolimus-eluting stent with biodegradable polymer improves clinical outcomes in patients with acute myocardial infarction
}

\author{
Yao-Jun Zhang, ${ }^{1,2}$ Javaid Iqbal, ${ }^{1}$ Stephan Windecker, ${ }^{3}$ Axel Linke, ${ }^{4}$ Diethmar Antoni, ${ }^{5}$ \\ Hae Young Sohn, ${ }^{6}$ Roberto Corti, ${ }^{7}$ Gerrit-Anne van Es, ${ }^{8}$ Samuel Copt, ${ }^{9}$ \\ Pedro Eerdmans, ${ }^{9}$ Rana Saitta, ${ }^{9}$ Marie-Claude Morice, ${ }^{10}$ Carlo Di Mario, ${ }^{11}$ \\ Peter Juni, ${ }^{12}$ William Wijns, ${ }^{13}$ Pawel Buszman, ${ }^{14}$ Patrick W Serruys ${ }^{1}$
}

- Additional material is published online only. To view please visit the journal online (http://dx.doi.org/10.1136/ heartjnl-2014-306359).

For numbered affiliations see end of article.

\section{Correspondence to} Professor Patrick W Serruys, Thoraxcenter, Erasmus Medical Center, PO Box 2125, Rotterdam 3000 CC, the Netherlands; patrick.w.j.c.serruys@gmail. com

Y-JZ and JI have contributed equally to this study.

Received 17 June 2014 Revised 29 September 2014 Accepted 26 October 2014 Published Online First 25 November 2014

CrossMark

\section{ABSTRACT}

Objective To investigate clinical outcomes of coronary intervention using a biolimus-eluting stent (BES) compared with a sirolimus-eluting stent (SES) in patients with acute myocardial infarction (AMI) in the Limus Eluted from $A$ Durable versus ERodable Stent (LEADERS) coating trial at the final 5-year follow-up.

Methods The LEADERS trial is a multicentre all-comer study, where patients $(n=1707)$ were randomised to percutaneous intervention with either BES containing biodegradable polymer or SES containing durable polymer. Out of 1707 patients enrolled in this trial, 573 patients had percutaneous coronary intervention for $\mathrm{AMI}$ $(B E S=280, S E S=293)$ and were included in the current analysis. Patient-oriented composite endpoint (POCE, including all death, all myocardial infarction (MI) and all revascularisations), major adverse cardiac events (MACE, including cardiac death, $\mathrm{Ml}$ and clinically indicated target vessel revascularisation) and stent thrombosis were assessed at 5-year follow-up.

Results The baseline clinical, angiographic and procedural characteristics were well matched between BES and SES groups. In all patients with AMI, coronary intervention with a BES, compared with SES, significantly reduced POCE (28.9\% vs $42.3 \%$; relative risk (RR) 0.61 , $95 \% \mathrm{Cl} 0.47$ to $0.82, p=0.001$ ) at 5 -year follow-up. There was also a reduction in MACE rate in the BES group (18.2\% vs $25.9 \%$; RR $0.67,95 \% \mathrm{Cl} 0.47$ to $0.95, p=0.025)$; however, there was no difference in cardiac death and stent thrombosis. In patients with ST-elevation MI (STEMI), coronary intervention with BES significantly reduced POCE (24.4\% vs $39.3 \%$; RR 0.55 , $95 \% \mathrm{Cl} 0.36$ to $0.85, \mathrm{p}=0.006), \operatorname{MACE}(12.6 \%$ vs $25.0 \%$; RR $0.47,95 \% \mathrm{Cl} 0.26$ to $0.83, \mathrm{p}=0.008$ ) and cardiac death (3.0\% vs $11.4 \%$; RR $0.25,95 \%$ Cl 0.08 to $0.75, p=0.007)$, along with a trend towards reduction in definite stent thrombosis (3.7\% vs $8.6 \%$; RR 0.41 , $95 \% \mathrm{Cl} 0.15$ to $1.18, p=0.088)$, compared with SES. Conclusions BES, compared with SES, significantly improved safety and efficacy outcomes in patients with AMI, especially those with STEMI, at 5-year follow-up. Trial registration number NCT 00389220.

\section{INTRODUCTION}

Patients with acute myocardial infarction (AMI), comprising ST-segment elevation myocardial infarction (STEMI) and non-STEMI (NSTEMI), are often treated with percutaneous coronary intervention (PCI). Clinical trials have confirmed the efficacy of PCI in the treatment of AMI. ${ }^{1-3}$ Furthermore, use of stents has been shown to reduce major adverse cardiac events (MACE) compared with balloon angioplasty alone. ${ }^{4}$ However, the choice of stent in patients undergoing PCI for AMI remains debatable as AMI is a risk factor for device-related adverse outcomes, including stent thrombosis. ${ }^{5}$

Bare metal stents (BMS) minimise the risk of acute closure and constrictive remodelling compared with balloon angioplasty, but are associated with restenosis due to neointimal hyperplasia. First-generation drug-eluting stents (DES) releasing sirolimus or paclitaxel from durable polymers reduce the need for repeat revascularisation, but delay vessel healing due to chronic inflammation induced by the presence of durable polymer. Meta-analyses have shown no significant difference in mortality, myocardial infarction (MI) and stent thrombosis at 1 year between BMS and first-generation DES, but have shown a significant reduction in target vessel revascularisation in the DES group. ${ }^{6}{ }^{7}$ Conversely, clinical studies and meta-analyses have also raised concerns about late and very late stent thrombosis with use of DES in AMI. ${ }^{8-11}$ Newer-generation DES with biocompatible or biodegradable polymers have been shown to have better safety and efficacy. ${ }^{12} 13$ In particular, DES with biodegradable polymers provide controlled drug release with subsequent degradation of the polymer rendering the stent surface more close to a BMS after the period of biodegradation. A few studies have compared the newer-generation DES against firstgeneration DES and reported conflicting outcomes at 1-3 years' follow-up. ${ }^{14}{ }^{15}$ Due to concerns about very late stent thrombosis, further follow-up data are warranted; however, to date, there are no longerterm (5-year) follow-up data for comparison of the newer-generation DES against first-generation DES in patients with AMI. Thus, we hypothesised that coronary intervention with a biodegradable polymer biolimus-eluting stent (BES) would improve clinical outcomes compared with intervention with a durable polymer sirolimus-eluting stent (SES) in AMI subpopulation of the 'all-comers' Limus Eluted from $A$ Durable versus ERodable Stent (LEADERS) coating trial. 


\section{Coronary artery disease}

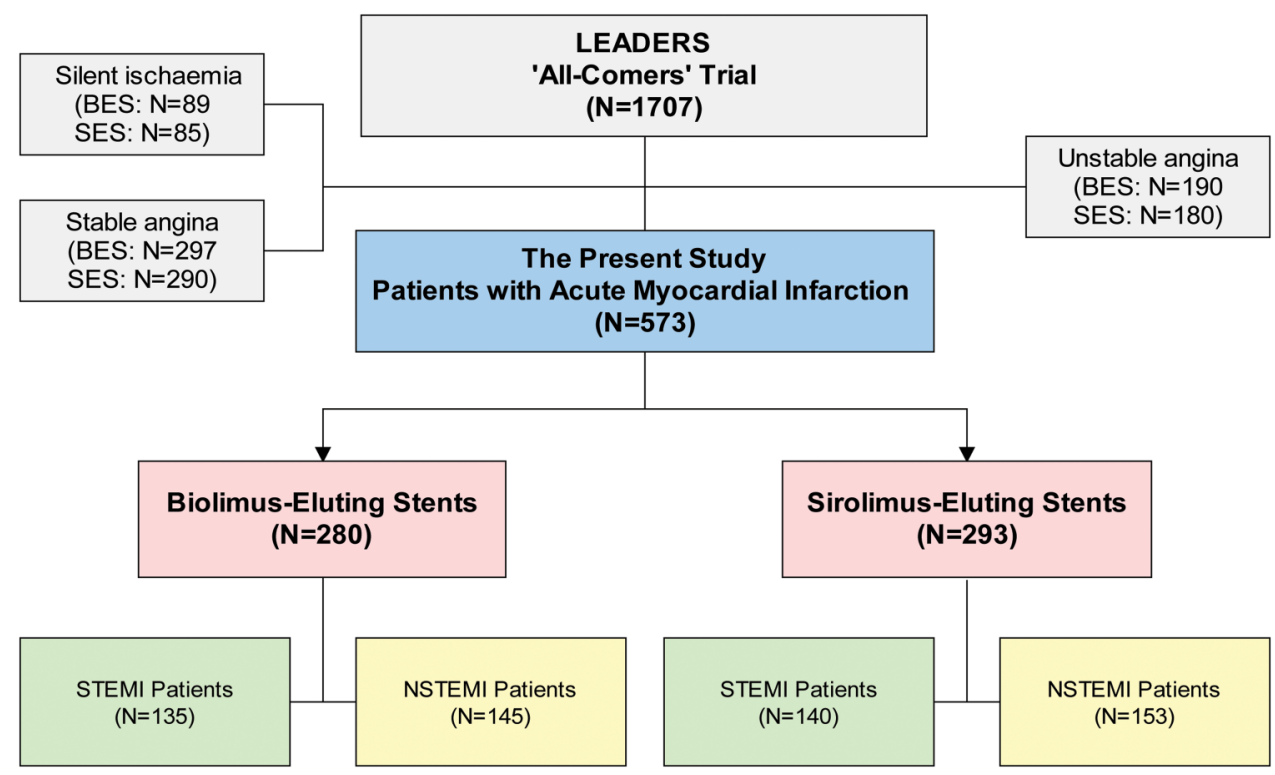

Figure 1 Study flow chart. BES, biolimus-eluting stent; LEADERS, Limus Eluted from A Durable versus ERodable Stent coating; NSTEMI, non-ST-segment elevation myocardial infarction; SES, sirolimus-eluting stent; STEMI, ST-segment elevation myocardial infarction.

Table 1 Baseline characteristics of acute myocardial infarction population stratified by stent type

\begin{tabular}{|c|c|c|c|}
\hline Characteristics & Biolimus-eluting stents $(n=280)$ & Sirolimus-eluting stents $(n=293)$ & p Value \\
\hline \multicolumn{4}{|l|}{ Patient } \\
\hline Age, years & $62.9 \pm 11.7$ & $62.8 \pm 11.7$ & 0.931 \\
\hline Men & $215(76.8 \%)$ & $210(71.7 \%)$ & 0.162 \\
\hline $\mathrm{BMI}, \mathrm{kg} / \mathrm{m}^{2}$ & $27.5 \pm 4.4$ & $27.8 \pm 4.6$ & 0.427 \\
\hline \multicolumn{4}{|l|}{ Cardiovascular risk factors } \\
\hline Diabetes mellitus & 55 (19.6\%) & $46(15.7 \%)$ & 0.216 \\
\hline Diabetes requiring insulin & $17(30.9 \%)$ & $20(43.5 \%)$ & 0.192 \\
\hline Hypertension & $181(64.6 \%)$ & $198(67.6 \%)$ & 0.458 \\
\hline Hypercholesterolaemia & $152(54.3 \%)$ & $176(60.1 \%)$ & 0.162 \\
\hline Current smoker & $107(38.2 \%)$ & $115(39.2 \%)$ & 0.799 \\
\hline Family history of CAD & $98(35 \%)$ & $115(39.2 \%)$ & 0.293 \\
\hline History of Ml & $61(21.8 \%)$ & $61(20.8 \%)$ & 0.778 \\
\hline History of $\mathrm{PCl}$ & $51(18.2 \%)$ & $51(17.4 \%)$ & 0.800 \\
\hline Previous CABG & $13(4.6 \%)$ & $14(4.8 \%)$ & 0.939 \\
\hline \multicolumn{4}{|l|}{ Clinical presentation } \\
\hline Non-ST-elevation MI & $145(51.8 \%)$ & $153(52.2 \%)$ & 0.918 \\
\hline ST-elevation MI (h) & $135(48.2 \%)$ & $140(47.8 \%)$ & 0.918 \\
\hline$<6$ & $92(68.1 \%)$ & $83(59.3 \%)$ & 0.127 \\
\hline$\geq 6-24$ & $26(19.3 \%)$ & $36(25.7 \%)$ & 0.200 \\
\hline$>24-72$ & $12(8.9 \%)$ & $16(11.4 \%)$ & 0.486 \\
\hline$>72$ & $5(3.7 \%)$ & $5(3.6 \%)$ & 0.953 \\
\hline LVEF, \% & $51.5 \pm 10.1$ & $51.4 \pm 11.8$ & 0.734 \\
\hline \multicolumn{4}{|l|}{ Lesion complexity } \\
\hline Multivessel disease & $69(24.6 \%)$ & $55(18.8 \%)$ & 0.088 \\
\hline Small-vessel disease (RVD <2.75 mm) & $180(64.3 \%)$ & $183(62.5 \%)$ & 0.650 \\
\hline Long lesions (>20 mm) & $94(33.6 \%)$ & $109(37.2 \%)$ & 0.364 \\
\hline Study lesions per patient & $1.5 \pm 0.8$ & $1.4 \pm 0.7$ & 0.076 \\
\hline One & $184(65.7 \%)$ & $213(72.7 \%)$ & 0.070 \\
\hline Two & $72(25.7 \%)$ & $59(20.1 \%)$ & 0.112 \\
\hline Three & $17(6.1 \%)$ & $17(5.8 \%)$ & 0.892 \\
\hline$>$ Four & $7(2.5 \%)$ & $4(1.4 \%)$ & 0.322 \\
\hline SYNTAX score (patient level) & $14.7 \pm 8.8$ & $15.3 \pm 8.7$ & 0.321 \\
\hline
\end{tabular}




\section{METHODS}

The LEADERS trial was conducted in accordance with the principles of the Declaration of Helsinki, and all site-specific Institutional Review Boards and applicable regulatory agencies approved the study protocol before study initiation.

\section{LEADERS trial}

Study design of the LEADERS trial (NCT 00389220) has been previously described ${ }^{16} 17$ and is outlined in figure 1 . Briefly, this was an all-comers prospective, multicentre, randomised, assessorblind, non-inferiority trial. Patients $(n=1707)$ with age $>18$ years and symptomatic coronary artery disease with $>50 \%$ stenosis in one or more native coronary arteries or saphenous vein bypass graft were included. Exclusion criteria were limited and included pregnancy, known intolerance to aspirin, clopidogrel, heparin, stainless steel, sirolimus, biolimus and contrast material, inability to provide informed consent, patient participation in another trial before reaching the primary endpoint or planned surgery within 6 months of PCI unless dual antiplatelet therapy was maintained throughout the perioperative period. Patients were 1:1 randomised to either BES (BioMatrix Flex, Biosensors Europe, Morges, Switzerland) or SES (Cypher Select, Cordis Corporation, Bridgewater, New Jersey, USA).
The current study included all patients with AMI (including STEMI and NSTEMI) in the LEADERS cohort. As an 'allcomers' trial, no limitation was placed on the number of treated lesions, number of vessels or lesion length according to the randomisation group. Patients were mandated to receive the same stent type for all lesions. All procedures were performed according to routine local clinical practice using standard techniques.

\section{Study endpoints}

The primary endpoint of the LEADERS trial was MACE, defined as composite of cardiac death, MI (Q-wave and non-Q-wave) or clinically indicated target vessel revascularisation within 9 months. For this substudy, we report MACE at 5 years and patient-oriented composite endpoint (POCE), a more comprehensive endpoint including all-cause death, all $\mathrm{MI}$ and any revascularisation that have been recommended by Academic Research Consortium (ARC) ${ }^{18}$ after commencement of the LEADERS trial. We have also included individual endpoints including cardiac mortality and stent thrombosis at 5-year follow-up. An independent clinical events committee blindly adjudicated all events. Patient safety was assessed at prespecified intervals by an independent data and safety monitoring board.

Table 2 Angiographic and procedural characteristics

\begin{tabular}{|c|c|c|c|}
\hline Characteristics & Biolimus-eluting stents $(n=409)$ & Sirolimus-eluting stents $(n=399)$ & p Value \\
\hline \multicolumn{4}{|l|}{ Baseline QCA results } \\
\hline $\mathrm{RVD}, \mathrm{mm}$ & $2.69 \pm 0.62$ & $2.65 \pm 0.58$ & 0.529 \\
\hline $\mathrm{MLD}, \mathrm{mm}$ & $0.77 \pm 0.55$ & $0.75 \pm 0.58$ & 0.733 \\
\hline Diameter stenosis, \% & $70.9 \pm 19.9$ & $70.9 \pm 21.9$ & 0.967 \\
\hline \multicolumn{4}{|l|}{ Procedure } \\
\hline Number of stent per lesion & $2.2 \pm 0.5$ & $2.2 \pm 0.6$ & 0.804 \\
\hline Total stent length per lesion & $26.6 \pm 15$ & $27.9 \pm 15.2$ & 0.201 \\
\hline Direct stenting & $129(31.9 \%)$ & $109(27.4 \%)$ & 0.185 \\
\hline Lesion success & $401(99.3 \%)$ & $379(97.9 \%)$ & 0.112 \\
\hline TIMI flow (preprocedure) & & & 0.891 \\
\hline 0 & $98(23.4 \%)$ & $102(25 \%)$ & \\
\hline 1 & $7(1.7 \%)$ & $14(3.4 \%)$ & \\
\hline 2 & $18(4.3 \%)$ & $29(7.1 \%)$ & \\
\hline 3 & $283(67.5 \%)$ & $247(60.5 \%)$ & \\
\hline TIMI flow (postprocedure) & & & 0.966 \\
\hline 0 & $2(0.5 \%)$ & $7(1.8 \%)$ & \\
\hline 1 & $0(0 \%)$ & $2(0.5 \%)$ & \\
\hline 2 & $6(1.5 \%)$ & $6(1.5 \%)$ & \\
\hline 3 & $400(97.8 \%)$ & $380(95.7 \%)$ & \\
\hline \multicolumn{4}{|l|}{ Postprocedural QCA results } \\
\hline RVD, mm & $2.78 \pm 0.49$ & $2.77 \pm 0.48$ & 0.640 \\
\hline MLD, mm & $2.34 \pm 0.47$ & $2.31 \pm 0.54$ & 0.254 \\
\hline Diameter stenosis, \% & $15.6 \pm 9.0$ & $16.4 \pm 13.9$ & 0.348 \\
\hline \multicolumn{4}{|l|}{ Cardiac enzymes* } \\
\hline \multicolumn{4}{|l|}{ 6-8 h postprocedure } \\
\hline Creatine kinase, U/L & $946 \pm 1407$ & $1081 \pm 1601$ & 0.641 \\
\hline Creatine kinase MB, U/L & $92 \pm 155$ & $108 \pm 175$ & 0.502 \\
\hline Troponin, ng/mL & $5.7 \pm 13.9$ & $7.9 \pm 23.1$ & 0.447 \\
\hline \multicolumn{4}{|l|}{$18 \mathrm{~h}$ postprocedure or discharge } \\
\hline Creatine kinase, U/L & $744 \pm 1301$ & $687 \pm 956$ & 0.676 \\
\hline Creatine kinase $\mathrm{MB}, \mathrm{U} / \mathrm{L}$ & $57 \pm 81$ & $61 \pm 86$ & 0.755 \\
\hline Troponin, ng/mL & $5.1 \pm 16.8$ & $4.7 \pm 11.0$ & 0.651 \\
\hline
\end{tabular}




\section{Statistical analysis}

Continuous variables are presented as mean $\pm \mathrm{SD}$, and categorical data as counts and percentages. Patients were analysed on an intention-to-treat analysis. Time-to-event variables are presented as Kaplan-Meier curves, and incidences compared using the log-rank test. All data were analysed using SAS V.9.3 (SAS Institute, Cary, North Carolina, USA).

\section{RESULTS}

\section{Patient characteristics}

Out of 1707 patients enrolled in the LEADERS trial, 573 patients were with AMI-298 with NSTEMI and 275 with STEMI. These patients were treated with either BES $(n=280)$ or SES $(n=293)$. Study flow chart is shown in figure 1.

Patients in the BES and SES groups were well-matched for baseline demographic, clinical and angiographic characteristics (table 1).

\section{Procedural and medication details}

A lesion-level comparison for angiographic and procedural parameters did not show any difference between the two treatment groups (table 2). There was also no difference in use of evidencebased medication in the BES and SES groups, especially the use of antiplatelet therapy throughout the 5-year follow-up (table 3). Dual antiplatelet therapy was mandated for 12 months postdevice implantation; however, only two-third of patients in each group were taking dual antiplatelet therapy at 1 year (BES 65.9\%

Table 3 Medication use up to 5-year follow-up

\begin{tabular}{|c|c|c|c|}
\hline Medications & $\begin{array}{l}\text { Biolimus-eluting } \\
\text { stents }(n=280)\end{array}$ & $\begin{array}{l}\text { Sirolimus-eluting } \\
\text { stents }(n=293)\end{array}$ & $\begin{array}{l}p \\
\text { Value }\end{array}$ \\
\hline \multicolumn{4}{|l|}{ During procedure } \\
\hline $\begin{array}{l}\text { Glycoprotein IIb/IIla } \\
\text { antagonists }\end{array}$ & $127(45.4 \%)$ & $118(40.3 \%)$ & 0.219 \\
\hline Loading dose clopidogrel & 235 (83.9\%) & $245(83.6 \%)$ & 0.920 \\
\hline $300 \mathrm{mg}$ & 35 (12.5\%) & $42(14.3 \%)$ & 0.520 \\
\hline $600 \mathrm{mg}$ & $185(66.1 \%)$ & $188(64.2 \%)$ & 0.632 \\
\hline Other & $15(5.3 \%)$ & $15(5.1 \%)$ & 0.791 \\
\hline \multicolumn{4}{|l|}{ At 1 month } \\
\hline Aspirin & $262(98.1 \%)$ & $270(98.9 \%)$ & 0.4568 \\
\hline Clopidogrel & $266(99.6 \%)$ & $266(97.4 \%)$ & 0.0352 \\
\hline DAPT & $262(98.1 \%)$ & $263(96.3 \%)$ & 0.2056 \\
\hline Statins & $254(90.71 \%)$ & $259(88.4 \%)$ & 0.3649 \\
\hline$\beta$-blockers & $243(86.79 \%)$ & $248(84.64 \%)$ & 0.4638 \\
\hline ACE inhibitors & 199 (71.07\%) & $216(73.72 \%)$ & 0.4782 \\
\hline \multicolumn{4}{|l|}{ At 1 year } \\
\hline Aspirin & $254(97.3 \%)$ & $260(95.6 \%)$ & 0.2816 \\
\hline Clopidogrel & $178(68.2 \%)$ & $169(62.1 \%)$ & 0.1418 \\
\hline DAPT & $172(65.9 \%)$ & $163(59.9 \%)$ & 0.1536 \\
\hline Statins & $243(86.79 \%)$ & $245(83.62 \%)$ & 0.2862 \\
\hline$\beta$-blockers & $228(81.43 \%)$ & $241(82.25 \%)$ & 0.7981 \\
\hline ACE inhibitors & $175(62.5 \%)$ & $190(64.85 \%)$ & 0.5593 \\
\hline \multicolumn{4}{|l|}{ At 5 years } \\
\hline Aspirin & $212(91 \%)$ & $221(93.6 \%)$ & 0.2798 \\
\hline Clopidogrel & $24(10.3 \%)$ & $25(10.6 \%)$ & 0.9174 \\
\hline DAPT & $17(7.3 \%)$ & $22(9.3 \%)$ & 0.4269 \\
\hline Statins & $204(72.86 \%)$ & $200(68.26 \%)$ & 0.2277 \\
\hline$\beta$-blockers & $195(69.64 \%)$ & $193(65.87 \%)$ & 0.3343 \\
\hline ACE inhibitors & $141(50.36 \%)$ & $142(48.46 \%)$ & 0.6505 \\
\hline
\end{tabular}

vs SES $59.9 \%, p=0.154)$ ). At 5 years, majority of patients in both groups were taking aspirin $(93.6 \%$ vs $91 \%, \mathrm{p}=0.280)$.

\section{Clinical outcomes}

All-cause mortality in the overall AMI population of LEADERS trial was $4.7 \%$ at 1 -year follow up and $13.3 \%$ at 5 -year follow-up (figure 2). Coronary intervention with a BES, compared with SES, significantly reduced POCE $(28.9 \%$ vs $42.3 \%$, relative risk (RR) $0.61,95 \% \mathrm{CI} 0.47$ to $0.82, \mathrm{p}=0.001$ ) at 5 -year follow-up (figure 2). Furthermore, MACE was also significantly lower in the BES group $(18.2 \%$ vs $25.9 \%$, RR 0.67 , $95 \%$ CI 0.47 to $0.95, \mathrm{p}=0.025$ ) at 5 -year follow-up (figure 3 ). However, there was no difference in individual endpoints of death, cardiac death, MI, target vessel revascularisation, target lesion revascularisation or stent thrombosis in the BES and SES groups (figure 2). There was a trend towards reduction in the risk of definite $(4.3 \%$ vs $6.8 \%$, RR $0.61,95 \%$ CI 0.30 to 1.25 , $\mathrm{p}=0.174)$ and definite/probable (5.4\% vs $8.5 \%$, HR $0.61,95 \%$ CI 0.32 to $1.16, p=0.128$ ) stent thrombosis with BES compared with SES (figure 2). The incidence of stent thrombosis according to ARC-defined time periods of acute, subacute, late and very late is shown in table 4. This benefit of BES on stent thrombosis was seen at both short-term and long-term follow-up as evident by the landmark analysis (figure 4).

\section{Stratified analysis according to AMI type}

Dividing AMI population further into STEMI and NSTEMI groups also revealed similar baseline characteristics among the two treatment arms (see online supplementary table S1). Angiographic and procedural characteristics of STEMI and NSTEMI subgroups are shown in online supplementary table S2. Five-year outcomes stratified by STEMI and NSTEMI population revealed that coronary intervention with BES improved POCE compared with intervention with SES in both AMI groups (see online supplementary table S3). In the STEMI group, cardiac death and MACE were significantly lower in the BES group compared with SES group; however, there was no difference in the NSTEMI group (figure 3). Furthermore, there was a trend towards reduction in definite $(3.7 \%$ vs $8.6 \%$, RR $0.41,95 \%$ CI 0.15 to $1.18, \mathrm{p}=0.088)$ and definite/probable ( $4.4 \%$ vs $10.0 \%$, RR $0.43,95 \%$ CI 0.16 to $1.11, \mathrm{p}=0.070$ ) stent thrombosis with use of BES in STEMI population (see online supplementary table S3).

\section{DISCUSSION}

This post hoc analysis of patients with AMI in the LEADERS trial has shown improved 5 -year clinical outcomes with coronary intervention using BES containing biodegradable polymer reducing POCE and MACE. Coronary intervention with a BES, compared with SES, was particularly beneficial in STEMI population, reducing POCE, MACE, cardiac death, repeat revascularisation and stent thrombosis.

The choice of stent in patients undergoing PCI for AMI has remained debatable. Coronary intervention with first-generation DES-eluting sirolimus or paclitaxel from a durable polymer has generally reduced need for revascularisation, but has shown no improvement in mortality compared with intervention with BMS. ${ }^{6}{ }^{19}{ }^{20}$ Furthermore, risk of late stent thrombosis with firstgeneration DES tends to offset benefit from reduction in revascularisation in patients with STEMI as seen in real-world registries, ${ }^{89}$ clinical trials ${ }^{21}$ and a recent meta-analysis of 15 clinical trials comparing BMS and first-generation DES. ${ }^{10}$

Newer-generation DES, eluting zotarolimus, everolimus or biolimus from biocompatible or biodegradable polymers, have been 


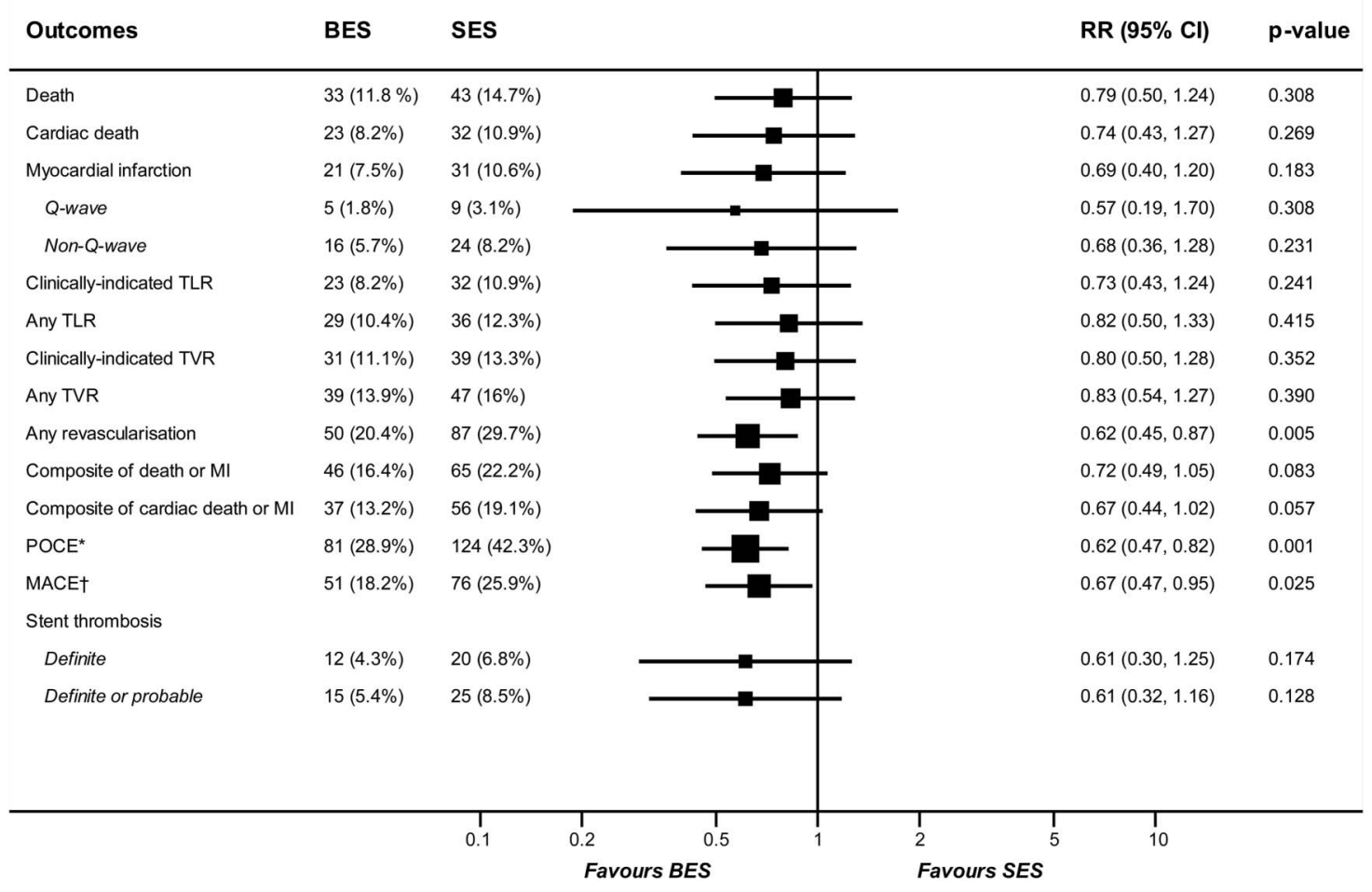

Figure 2 Clinical outcomes in patients with acute myocardial infarction at 5-year follow-up. *Patient-oriented composite endpoint (all-cause death, $\mathrm{Ml}$, all-cause revascularisation). tComposite of cardiac death, $\mathrm{MI}$ and clinically indicated target vessel revascularisation. BES, biolimus-eluting stent; MACE, major adverse cardiac event; MI, myocardial infarction; POCE, patient-oriented composite endpoint; RR, relative risk; SES, sirolimus-eluting stent; TLR, target lesion revascularisation; TVR, target vessel revascularisation.

shown to offer better safety outcomes. The EXAMINATION (clinical Evaluation of the Xience-V stent in Acute Myocardial INfArcTION) trial compared the durable polymer

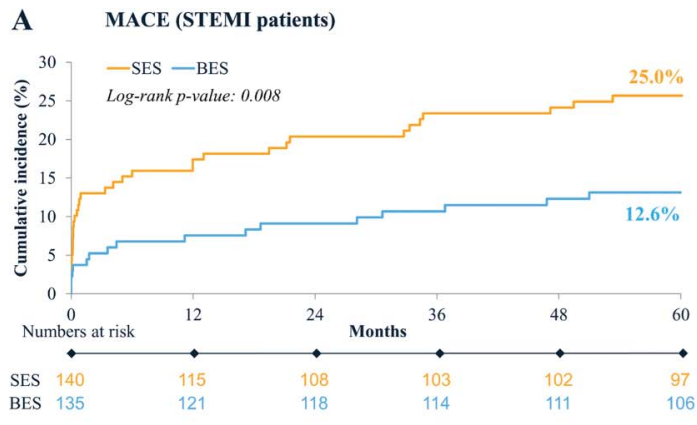

\section{B MACE (NSTEMI patients)}

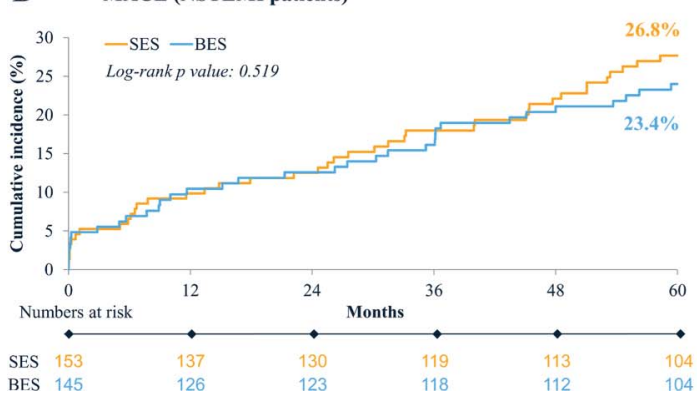

everolimus-eluting stent with BMS in patients with STEMI and showed reduction in target lesion revascularisation $(3.7 \%$ vs $6.8 \%$, $\mathrm{p}=0.008$ ) but no difference in the composite endpoint of all-cause
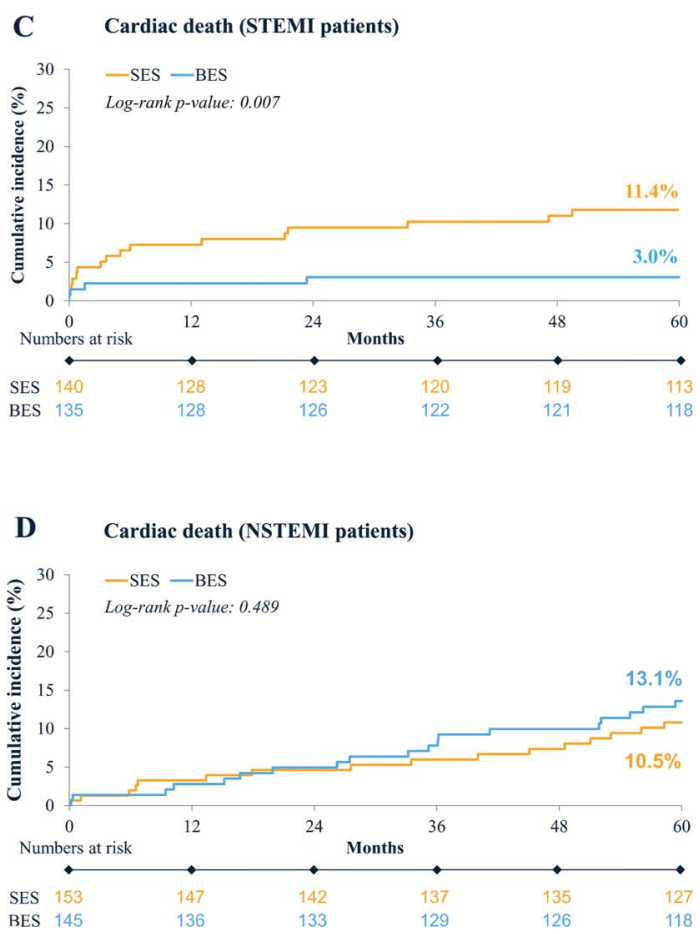

Figure 3 Kaplan-Meier curves for 5-year cardiac death and MACE. MACE and cardiac death were significantly lower in BES group in patients with STEMI (A, C). No differences were detected statistically on MACE and cardiac death between the 2 groups in patients with NSTEMI (B, D). BES, biolimus-eluting stent; MACE, major adverse cardiac event; NSTEMI, non-ST-segment elevation myocardial infarction; SES, sirolimus-eluting stent; STEMI, ST-elevation myocardial infarction. 
Table 4 Stent thrombosis for patients with acute myocardial infarction by time period

\begin{tabular}{llll}
\hline Stent thrombosis & $\begin{array}{l}\text { Biolimus-eluting } \\
\text { stents }(\mathbf{n}=\mathbf{2 8 0})\end{array}$ & $\begin{array}{l}\text { Sirolimus-eluting } \\
\text { stents }(\mathbf{n}=293)\end{array}$ & $\begin{array}{l}\mathbf{p} \\
\text { Value }\end{array}$ \\
\hline Early (0-30 days) & & & \\
$\quad$ Definite ST & $6(2.1 \%)$ & $11(3.8 \%)$ & 0.254 \\
$\quad$ Definite or probable ST & $7(2.5 \%)$ & $13(4.4 \%)$ & 0.206 \\
$\begin{array}{l}\text { Late (31-360 days) } \\
\quad\end{array}$ & $2(0.7 \%)$ & $2(0.7 \%)$ & 0.990 \\
$\quad$ Definite ST & $2(0.7 \%)$ & $2(0.7 \%)$ & 0.990 \\
$\quad$ Definite or probable ST & & \\
Very late (361-1800 days) & & $7(2.7 \%)$ & 0.364 \\
$\quad \begin{array}{l}\text { Definite ST } \\
\text { Definite or probable ST }\end{array}$ & $6(2.3 \%)$ & $10(3.8 \%)$ & 0.318 \\
\hline ST, stent thrombosis. & & &
\end{tabular}

death, MI and target lesion revascularisation (11.9\% vs $14.2 \%$, $\mathrm{p}=0.19$ ) at 1 -year follow-up. ${ }^{12}$ The COMFORTABLE-AMI (Comparison of Biolimus Eluted From an Erodible Stent Coating With Bare Metal Stents in Acute ST-Elevation Myocardial Infarction) trial, comparing BES against BMS, demonstrated the superiority of the biodegradable polymer BES to BMS with the identical metallic platform in terms of a significant reduction in the primary endpoint of MACE defined as the composite of cardiac death, target vessel-related $\mathrm{MI}$ and ischaemia-driven target lesion revascularisation $(4.3 \%$ vs $8.7 \%, \mathrm{p}=0.004)$ and POCE $(8.4 \%$ vs $12.2 \%, \mathrm{p}=0.04)$ at 1 -year follow-up. ${ }^{13}$ Definite stent thrombosis was numerically lower in the BES group $(0.9 \%$ vs $2.1 \%, \mathrm{p}=0.10)$, and there was no difference in mortality $(2.9 \% \mathrm{vs}$ $3.5 \%, \mathrm{p}=0.53) .{ }^{13}$ Two-year follow-up results were recently reported, showing persistent benefit of BES over BMS. ${ }^{22}$ A pooled analysis of the EXAMINATION and COMFORTABLE-AMI trials also showed that newer-generation DES improve safety and efficacy compared with BMS at 1-year follow-up. ${ }^{23}$ Further follow-up is awaited to evaluate the long-term impact of durable polymer newer-generation DES on very late stent thrombosis and its associated clinical impact.

Previous studies comparing zotarolimus-eluting and everolimus-eluting second-generation stents have not been able to show a convincing superiority over the first-generation DES in patients with AMI. ZEST-AMI compared the efficacy and safety of zotarolimus-eluting stents $(\mathrm{n}=108)$ against first-generation SES $(\mathrm{n}=110)$ and paclitaxel-eluting stents $(\mathrm{n}=110)$ in patients with STEMI. At 12 months, cumulative incidence rates of primary endpoint (MACE, composite of death, MI and ischaemia-driven target vessel revascularisation) in the zotarolimus-eluting, sirolimus-eluting and paclitaxel-eluting stents were $11.3 \%, 8.2 \%$ and
8.2\%, respectively $(\mathrm{p}=0.834) .{ }^{15}$ XAMI (XienceV Stent vs. Cypher Stent in Primary PCI for Acute Myocardial Infarction) trial has compared everolimus-eluting stent against the firstgeneration SES and reported a lower rate of MACE (composite of cardiac death, AMI or any target vessel revascularisation) with everolimus-eluting stent $(4.0 \%$ vs $7.7 \%, \mathrm{p}=0.048)$ but no significant difference in cardiac mortality $(1.5 \%$ vs $2.7 \%, \mathrm{p}=0.36)$ or the incidence of definite/probable stent thrombosis $(1.2 \%$ vs $2.7 \%, \mathrm{p}=0.21$ ) at 1 year. ${ }^{14}$ However, at 3 -year follow-up, there was no difference in everolimus-eluting stent and SES groups for MACE (everolimus-eluting stent $8.0 \%$ vs SES $10.5 \%, \mathrm{p}=0.30$ ), cardiac death $(2.5 \%$ vs $2.7 \%, \mathrm{p}=0.86)$ and definite/probable stent thrombosis $(2.3 \%$ vs $3.2 \%, \mathrm{p}=0.60)$. AMI substudies of other trials comparing everolimus-eluting stent against SES, including Basket-PROVE (the BASKET-Prospective Validation Examination), ${ }^{24}$ EXCELLENT (Efficacy of Xience/Promus Versus Cypher to Reduce Late Loss After Stenting) ${ }^{25}$ and SORT-OUT IV (The Scandinavian Organization for Randomized Trials with Clinical Outcome IV), ${ }^{26}$ have also showed no significant advantage of everolimus-eluting stent over SES in patients with AMI or STEMI. Our study, for the first time, has shown that BES with biodegradable polymer, compared with the durable polymer SES, reduced cardiac death as well as stent thrombosis in STEMI population at 5 -year follow-up.

The main strengths of the current study, compared with previous reports of newer-generation DES in AMI population, include having an 'all-comers' AMI population and the longest available (5-year) follow-up. Due to an 'all-comer' design, this study included patients with STEMI with complex anatomical disease $(22 \%$ patients had multivessel disease, $38 \%$ patients had lesions $>20 \mathrm{~mm}$ ). This may explain a slightly higher incidence of adverse events in both arms of study compared with other reports. The sample size in this study is moderate; however, due to longer follow-up, we were able to demonstrate a beneficial effect of BES in reducing cardiac death, which was not evident at 1-year follow-up of the current study as well as in the COMFORTABLE-AMI trial. The long-term follow-up also provides additional insights into late stent thrombosis, which is largely blamed on durable polymer. ${ }^{27}$ Undoubtedly, stent thrombosis is multifactorial in origin; patient-related, procedurerelated or device-related factors may play a role. ${ }^{27}$ Our results indicate that patients with AMI remain at a significant risk of late stent thrombosis even in the BES group (incidence of late stent thrombosis in the BES group: non-AMI 0.3\%, STEMI 1.6\%, NSTEMI 3.0\%). ${ }^{28}$ Therefore, adhering to an evidencebased medical therapy, including antiplatelet drugs, is vital in these high-risk patients. Furthermore, the dual antiplatelet regimen in the LEADERS trial included aspirin and clopidogrel.
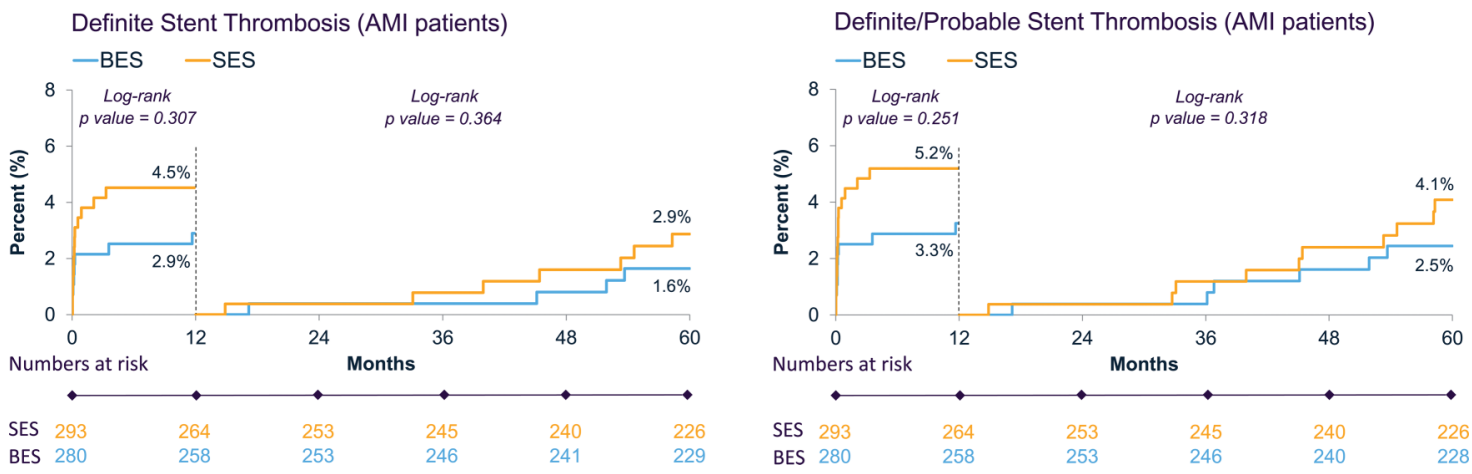

Figure 4 Kaplan-Meier curves for stent thrombosis with landmark analysis. AMI, acute myocardial infarction; BES, biolimus-eluting stent; SES, sirolimus-eluting stent. 
However, newer $\mathrm{P}_{2} \mathrm{Y}_{12}$ inhibitors (prasugrel and ticagrelor) with better clinical outcomes have largely replaced clopidogrel as part of dual antiplatelet therapy. ${ }^{29} 30$

This study has several limitations. It is a post hoc analysis of the data, and results should be viewed with caution. Some baseline characteristics, including ischaemia time, door-to-balloon time and thrombectomy use, were unavailable in both of the studied groups. The trial was not powered for stent thrombosis in various subgroups; therefore, possibility of a type I statistical error cannot be excluded. Type I error was also not corrected for multiple comparison. However, stent thrombosis according to the definitions of ARC was a prespecified endpoint and was adjudicated by an independent clinical events committee, and the incidence of definite stent thrombosis continued to diverge between the two investigated devices up to 5 years, which would make the play of chance unlikely.

\section{CONCLUSIONS}

The newer-generation BES with biodegradable polymer compared with SES with durable polymer provided significant improvements in clinical outcomes in patients with AMI at 5-year follow-up. The benefit appeared more significant in the subgroup of patients with STEMI. Our results suggest that BES should be favourably considered for treating patients with STEMI. However, long-term follow-up of the COMFORTABLE-AMI trial and further larger studies are needed to confirm our findings.

\section{Key messages}

\section{What is already known on this subject?}

Clinical trials have confirmed the efficacy of percutaneous coronary intervention (PCI) in the treatment of acute myocardial infarction. Use of stents has been shown to reduce major adverse cardiac events compared with balloon angioplasty alone. However, the choice of stent in patients undergoing $\mathrm{PCl}$ for acute myocardial infarction remains debatable.

\section{What might this study add?}

This study highlights that patients with acute myocardial infarction have shown improved 5-year clinical outcomes with biolimus-eluting stent (BES) containing biodegradable polymer reducing major cardiac adverse events (absolute risk reduction: 7.7\%). Coronary intervention with a BES, compared with a sirolimus-eluting stent (SES), was particularly beneficial in ST-elevation myocardial infarction (STEMI) population.

\section{How might this impact on clinical practice?}

This study, for the first time, has shown that coronary intervention with a biodegradable polymer BES, compared with the durable polymer SES, reduced cardiac death as well as stent thrombosis in STEMI population at 5-year follow-up; hence, use of the newer generation biodegradable polymer BES should be recommended in patients with STEMI.

\footnotetext{
Author affiliations

${ }^{1}$ Thoraxcenter, Erasmus Medical Center, Rotterdam, The Netherlands ${ }^{2}$ Nanjing Medical University, Nanjing First Hospital, Nanjing, China ${ }^{3}$ Department of Cardiology, University of Bern, Bern, Switzerland ${ }^{4}$ Herzzentrum Leipzig, Leipzig, Germany ${ }^{5}$ Department of Cardiology, Hospital Bogenhausen, Munich, Germany ${ }^{6}$ Department of Cardiology, University Hospital Munich, Munich, Germany ${ }^{7}$ HerzKlinik Hirslanden, Zürich, Switzerland

${ }^{8}$ Cardialysis BV, Rotterdam, The Netherlands
}

${ }^{9}$ Biosensors Europe SA, Morges, Switzerland

${ }^{10}$ Institut Cardiovasculaire Paris-Sud, Institut Hospitalier Jacques-Cartier, Massy, France

${ }^{11}$ NIHR Cardiovascular Biomedical Research Unit, Royal Brompton Hospital, London, UK

${ }^{12}$ CTU, University of Bern, Bern, Switzerland

${ }^{13}$ Department of Cardiology, Onze Lieve Vrouw Ziekenhuis, Aalst, Belgium ${ }^{14}$ American Heart of Poland, Ustroń, Poland

Contributors $Y-J Z, J$ and PWS designed and planned the study, interpreted the data and drafted the manuscript. YJZ, JI and SC merged and analysed the data. The other authors revised it critically for important intellectual content and gave the final approval of the version to be published.

Funding Biosensors Europe SA, Morges, Switzerland, sponsored this study.

Competing interests $\mathrm{FE}$ has received an institutional grant from Biosensors and consulting fees from Cordis. WW has received institutional research grants from Biosensors and Cordis, and was co-chairman of Cordis international advisory board (until May 2011). CDM has received a research grant to the institution from Biosensors. PE, SC and RS are employees of Biosensors Europe. BM has received research grants to the institution from Abbott and Cordis. SW has received research contracts to the institution from Abbott, Boston Scientific, Biosensors, Cordis and Medtronic. PJ is an unpaid steering committee or statistical executive committee member of trials funded by Abbott Vascular, Biosensors, Medtronic, and Johnson \& Johnson. The other authors report no conflicts of interest.

\section{Patient consent Obtained.}

Ethics approval All studies complied with the Declaration of Helsinki and were approved by the ethical review board of the institution involved, whereas the included patients provided written informed consent for participation in these studies.

Provenance and peer review Not commissioned; externally peer reviewed.

\section{REFERENCES}

1 Mehta SR, Cannon CP, Fox KA, et al. Routine vs selective invasive strategies in patients with acute coronary syndromes: a collaborative meta-analysis of randomized trials. JAMA 2005;293:2908-17.

2 Fox KA, Clayton TC, Damman P, et al. Long-term outcome of a routine versus selective invasive strategy in patients with non-ST-segment elevation acute coronary syndrome a meta-analysis of individual patient data. J Am Coll Cardiol 2010;55:2435-45.

3 Keeley EC, Boura JA, Grines CL. Primary angioplasty versus intravenous thrombolytic therapy for acute myocardial infarction: a quantitative review of 23 randomised trials. Lancet 2003;361:13-20.

4 Stone GW, Grines CL, Cox DA, et al. Comparison of angioplasty with stenting, with or without abciximab, in acute myocardial infarction. N Engl J Med 2002:346:957-66.

5 Iqbal J, Sumaya $W$, Tatman $V$, et al. Incidence and predictors of stent thrombosis: a single-centre study of 5,833 consecutive patients undergoing coronary artery stenting. Eurolntervention 2013;9:62-9.

6 Brar SS, Leon MB, Stone GW, et al. Use of drug-eluting stents in acute myocardial infarction: a systematic review and meta-analysis. J Am Coll Cardiol 2009:53:1677-89.

7 Palmerini T, Biondi-Zoccai G, Della Riva D, et al. Clinical outcomes with drug-eluting and bare-metal stents in patients with ST-segment elevation myocardial infarction: evidence from a comprehensive network meta-analysis. J Am Coll Cardiol 2013;62:496-504.

8 Steg PG, Fox KA, Eagle KA, et al. Mortality following placement of drug-eluting and bare-metal stents for ST-segment elevation acute myocardial infarction in the Global Registry of Acute Coronary Events. Eur Heart J 2009;30:321-9.

9 Daemen J, Tanimoto S, Garcia-Garcia HM, et al. Comparison of three-year clinical outcome of sirolimus- and paclitaxel-eluting stents versus bare metal stents in patients with ST-segment elevation myocardial infarction (from the RESEARCH and T-SEARCH Registries). Am J Cardiol 2007;99:1027-32.

10 Kalesan B, Pilgrim T, Heinimann K, et al. Comparison of drug-eluting stents with bare metal stents in patients with ST-segment elevation myocardial infarction. Eur Heart J 2012;33:977-87.

11 De Luca G, Dirksen MT, Spaulding C, et al. Drug-eluting vs bare-metal stents in primary angioplasty: a pooled patient-level meta-analysis of randomized trials. Arch Intern Med 2012;172:611-21; discussion 21-2.

12 Sabate $M$, Cequier $A$, Iniguez $A$, et al. Everolimus-eluting stent versus bare-metal stent in ST-segment elevation myocardial infarction (EXAMINATION): 1 year results of a randomised controlled trial. Lancet 2012;380:1482-90.

13 Raber L, Kelbaek H, Ostojic M, et al. Effect of biolimus-eluting stents with biodegradable polymer vs bare-metal stents on cardiovascular events among patients with acute myocardial infarction: the COMFORTABLE AMI randomized trial. JAMA 2012;308:777-87 
14 Hofma SH, Brouwer J, Velders MA, et al. Second-generation everolimus-eluting stents versus first-generation sirolimus-eluting stents in acute myocardial infarction. 1 -year results of the randomized XAMI (XienceV Stent vs. Cypher Stent in Primary PCI for Acute Myocardial Infarction) trial. J Am Coll Cardiol 2012;60:381-7.

15 Lee CW, Park DW, Lee SH, et al. Comparison of the efficacy and safety of zotarolimus-, sirolimus-, and paclitaxel-eluting stents in patients with ST-elevation myocardial infarction. Am J Cardiol 2009;104:1370-6.

16 Windecker S, Serruys PW, Wandel S, et al. Biolimus-eluting stent with biodegradable polymer versus sirolimus-eluting stent with durable polymer for coronary revascularisation (LEADERS): a randomised non-inferiority trial. Lancet 2008;372:1163-73.

17 Stefanini GG, Kalesan B, Serruys PW, et al. Long-term clinical outcomes of biodegradable polymer biolimus-eluting stents versus durable polymer sirolimus-eluting stents in patients with coronary artery disease (LEADERS): 4 year follow-up of a randomised non-inferiority trial. Lancet 2011;378:1940-8.

18 Cutlip DE, Windecker S, Mehran R, et al. Clinical end points in coronary stent trials: a case for standardized definitions. Circulation 2007;115:2344-51.

19 Spaulding $C$, Henry $P$, Teiger $E$, et al. Sirolimus-eluting versus uncoated stents in acute myocardial infarction. N Engl J Med 2006;355:1093-104.

20 Stone GW, Lansky AJ, Pocock SJ, et al. Paclitaxel-eluting stents versus bare-metal stents in acute myocardial infarction. N Engl J Med 2009;360:1946-59.

21 Holmvang L, Kelbaek H, Kaltoft A, et al. Long-term outcome after drug-eluting versus bare-metal stent implantation in patients with ST-segment elevation myocardial infarction: 5 years follow-up from the randomized DEDICATION trial (Drug Elution and Distal Protection in Acute Myocardial Infarction). JACC CardiovasC Interv 2013;6:548-53.

22 Raber L, Kelbaek H, Taniwaki M, et al. Biolimus-eluting stents with biodegradable polymer versus bare-metal stents in acute myocardial infarction: two-year clinical results of the COMFORTABLE AMI trial. Circ Cardiovasc Interv 2014;7:355-64.
23 Sabate M, Raber L, Heg D, et al. Comparison of newer-generation drug-eluting with bare-metal stents in patients with acute ST-segment elevation myocardial infarction: a pooled analysis of the EXAMINATION (clinical Evaluation of the Xience-V stent in Acute Myocardial INfArcTION) and COMFORTABLE-AMI (Comparison of Biolimus Eluted From an Erodible Stent Coating With Bare Metal Stents in Acute ST-Elevation Myocardial Infarction) trials. JACC Cardiovasc Interv 2014;7:55-63.

24 Pedersen SH, Pfisterer M, Kaiser C, et al. Drug-eluting stents and bare metal stents in patients with NSTE-ACS: 2-year outcome from the randomised BASKET-PROVE trial. Eurolntervention 2014;10:58-64.

25 Park KW, Chae IH, Lim DS, et al. Everolimus-eluting versus sirolimus-eluting stents in patients undergoing percutaneous coronary intervention: the EXCELLENT (Efficacy of Xience/Promus Versus Cypher to Reduce Late Loss After Stenting) randomized trial. J Am Coll Cardiol 2011;58:1844-54.

26 Antonsen L, Thayssen P, Hansen HS, et al. Outcomes after revascularisation with everolimus- and sirolimus-eluting stents in patients with acute coronary syndromes and stable angina pectoris: a substudy of the SORT OUT IV trial. Eurolntervention 2014;10:212-23.

27 Lüscher TF, Steffel J, Eberli FR, et al. Drug-eluting stent and coronary thrombosis. Circulation 2007;115:1051-8.

28 Serruys PW, Farooq V, Kalesan B, et al. Improved safety and reduction in stent thrombosis associated with biodegradable polymer-based biolimus-eluting stents versus durable polymer-based sirolimus-eluting stents in patients with coronary artery disease: final 5-year report of the LEADERS (Limus Eluted From A Durable Versus ERodable Stent Coating) randomized, noninferiority trial. JACC CardiovasC Interv 2013;6:777-89.

29 Wiviott SD, Braunwald E, McCabe CH, et al. Prasugrel versus clopidogrel in patients with acute coronary syndromes. N Engl J Med 2007;357:2001-15.

30 Wallentin L, Becker RC, Budaj A, et al. Ticagrelor versus clopidogrel in patients with acute coronary syndromes. N Engl J Med 2009;361:1045-57. 


\section{Heart}

\section{Biolimus-eluting stent with biodegradable polymer improves clinical outcomes in patients with acute myocardial infarction}

Yao-Jun Zhang, Javaid Iqbal, Stephan Windecker, Axel Linke, Diethmar Antoni, Hae Young Sohn, Roberto Corti, Gerrit-Anne van Es, Samuel Copt, Pedro Eerdmans, Rana Saitta, Marie-Claude Morice, Carlo Di Mario, Peter Juni, William Wijns, Pawel Buszman and Patrick W Serruys

Heart 2015 101: 271-278 originally published online November 25, 2014 doi: 10.1136/heartjnl-2014-306359

Updated information and services can be found at:

http://heart.bmj.com/content/101/4/271

These include:

Supplementary Material

References

Email alerting service
Supplementary material can be found at:

http://heart.bmj.com/content/suppl/2014/11/25/heartjnl-2014-306359. DC1

This article cites 30 articles, 5 of which you can access for free at: http://heart.bmj.com/content/101/4/271\#BIBL

Receive free email alerts when new articles cite this article. Sign up in the box at the top right corner of the online article.

Topic
Collections

Articles on similar topics can be found in the following collections

Interventional cardiology (2933)

Drugs: cardiovascular system (8842)

Acute coronary syndromes (2742)

Venous thromboembolism (495)

Clinical diagnostic tests (4779)

Percutaneous intervention (964)

\section{Notes}

To request permissions go to:

http://group.bmj.com/group/rights-licensing/permissions

To order reprints go to:

http://journals.bmj.com/cgi/reprintform

To subscribe to BMJ go to:

http://group.bmj.com/subscribe/ 